\title{
A separator theorem for string graphs and its applications
}

\author{
Jacob Fox* János Pach ${ }^{\dagger}$
}

\begin{abstract}
A string graph is the intersection graph of a collection of continuous arcs in the plane. It is shown that any string graph with $m$ edges can be separated into two parts of roughly equal size by the removal of $O\left(m^{3 / 4} \sqrt{\log m}\right)$ vertices. This result is then used to deduce that every string graph with $n$ vertices and no complete bipartite subgraph $K_{t, t}$ has at most $c_{t} n$ edges, where $c_{t}$ is a constant depending only on $t$. Another application shows that locally tree-like string graphs are globally tree-like: for any $\epsilon>0$, there is an integer $g(\epsilon)$ such that every string graph with $n$ vertices and girth at least $g(\epsilon)$ has at most $(1+\epsilon) n$ edges.
\end{abstract}

\section{Introduction}

A large part of computational geometry deals with representation and manipulation of various geometric objects. Special attention is paid to pairs of objects that are in contact with each other: detecting intersections among line segments, for example, belongs to the oldest and best studied chapter of computational geometry, already addressed in the first monograph devoted to the subject [31]. Yet, even in the special case of segments, little is known about elementary structural properties of the arising intersection patterns. The recognition of such intersection patterns (intersection graphs) is known to be NP-hard [20], [21].

Given a collection $C=\left\{\gamma_{1}, \ldots, \gamma_{n}\right\}$ of arcwise connected sets in the plane, their intersection graph $G=G(C)$ is a graph on the vertex set $C$, where $\gamma_{i}$ and $\gamma_{j}(i \neq j)$ are connected by an edge if and only if $\gamma_{i} \cap \gamma_{j} \neq \emptyset$. It is easy to show that every such intersection graph can be obtained as an intersection graph of a collection of (simple) continuous curves in the plane. Therefore, the intersection graphs of arcwise connected sets in the plane are often called string graphs.

Given a graph $G=(V, E)$ with vertex set $V$ and edge set $E$, a weight function $w: V \rightarrow \mathbb{R}_{\geq 0}$ is a nonnegative function on the vertex set such that the sum of the weights is at most 1 . The weight of a subset $S \subseteq V$, denoted by $w(S)$, is defined as $\sum_{v \in S} w(v)$.

A separator in a graph $G=(V, E)$ with respect to a weight function $w$ is a subset $S \subseteq V$ for which there is a partition $V=S \cup V_{1} \cup V_{2}$ such that $w\left(V_{1}\right), w\left(V_{2}\right) \leq 2 / 3$ and there is no edge between $V_{1}$ and $V_{2}$. If the weight function is not specified, it is assumed that $w(v)=\frac{1}{|V|}$ for every vertex $v \in V$.

\footnotetext{
*Department of Mathematics, Princeton, Princeton, NJ. Email: jacobfox@math.princeton.edu. Research supported by an NSF Graduate Research Fellowship and a Princeton Centennial Fellowship.

${ }^{\dagger}$ City College, CUNY and Courant Institute, NYU, New York, NY. Email: pach@cims.nyu.edu. Supported by NSF Grant CCF-05-14079, and by grants from NSA, PSC-CUNY, the Hungarian Research Foundation OTKA, and BSF.
} 
The Lipton-Tarjan separator theorem [24] states that for every planar graph $G$ with $n$ vertices and for every weight function $w$ for $G$, there is a separator of size $O\left(n^{1 / 2}\right)$. This has been generalized in various directions: to graphs embedded in a surface of bounded genus [15], graphs with a forbidden minor [1], intersection graphs of balls in $\mathbb{R}^{d}$ [26], intersection graphs of Jordan regions [10], and intersection graphs of convex sets in the plane [10]. Our main result is a separator theorem for string graphs.

Theorem 1.1 For every string graph $G$ with $m$ edges and for every weight function $w$ for $G$, there is a separator of size $O\left(m^{3 / 4} \sqrt{\log m}\right)$ with respect to $w$.

We do not believe that the bound on the separator size in Theorem 1.1 is tight. In fact, as in [13], we make the following conjecture.

Conjecture 1.2 Every string graph with $m$ edges has a separator of size $O(\sqrt{m})$.

This conjecture is known to be true in several special cases: (1) for intersection graphs of convex sets in the plane with bounded clique number [10], (2) for intersection graphs of curves, any pair of which have a bounded number of intersection points [10], and (3) for outerstring graphs, that is, intersection graphs of collections $C$ of curves with the property that there is a suitable curve $\gamma$ such that each member of $C$ has one endpoint on $\gamma$, but is otherwise disjoint from it [11].

Separator theorems have many important applications (see, e.g., [23] and [25]). Despite the apparent weakness of the bound in Theorem 1.1, it is still strong enough to yield some interesting corollaries.

For any graph $H$, a graph $G$ is called $H$-free if it does not have a (not necessarily induced) subgraph isomorphic to $H$. Given $H$ and a positive integer $n$, the extremal number $\operatorname{ex}(H, n)$ is defined as the maximum number of edges over all $H$-free graphs on $n$ vertices. The study of this parameter is a classical area of Turán type extremal graph theory; see [3]. The problem of investigating the same maximum restricted to intersection graphs of arcwise connected sets, convex bodies, segments, etc., was initiated in [29]. For partial results in this directions, see [29], [32], [10].

In the present paper, we use Theorem 1.1 to prove that for any bipartite graph $H$, there is a constant $c_{H}$ such that every $H$-free intersection graph of $n$ arcwise connected sets in the plane has at most $c_{H} n$ edges. Clearly, it is sufficient to prove this statement for balanced complete bipartite graphs $H=K_{t, t}$, as every bipartite graph with $t$ vertices is a subgraph of $K_{t, t}$.

Theorem 1.3 For any positive integer $t$, every $K_{t, t}$-free string graph with $n$ vertices has at most $t^{c \log \log t} n$ edges, where $c$ is an absolute constant.

A graph $G$ is called $d$-degenerate if every subgraph of $G$ has a vertex of degree at most $d$. Every $d$-degenerate graph has chromatic number at most $d+1$. Theorem 1.3 implies that every $K_{t, t}-$ free intersection graph of arcwise connected sets in the plane is $2 t^{c \log \log t}$-degenerate. Thus, we obtain

Corollary 1.4 For any positive integer $t$, the chromatic number of every $K_{t, t}$-free intersection graph of $n$ arcwise connected sets in the plane is at most $2 t^{c \log \log t}+1$. 
In [10], it was shown that every $K_{t, t}$-free intersection graphs of $n$ curves, no pair of which has more than a fixed constant number of points in common, has at most $c_{t} n$ edges, where the dependence on $t$ is exponential. In this case, our separator based approach gives a tight bound. In Section 5, we establish

Theorem 1.5 Let $k$ and $t$ be positive integers. There exists a constant $C_{k}$ depending only on $k$, such that the maximum number of edges of any $K_{t, t}$-free intersection graph $G$ of $n$ curves in the plane, no pair of which have more than $k$ points in common, is at most $C_{k} t n$. Apart from the value of the constant $C_{k}$, this bound cannot be improved.

A collection of curves in the plane is called a collection of pseudo-segments if no two of them have more than one point in common. The girth of a graph is the length of its shortest cycle. Kostochka and Nešetřil [18] proved that for any $\epsilon>0$, there is a positive integer $g(\epsilon)$ such that the intersection graph of any collection of pseudo-segments with girth at least $g(\epsilon)$ has at most $(1+\epsilon) n$ edges. Using our separator theorem, Theorem 1.1, this statement can be extended to all string graphs.

Theorem 1.6 For any $\epsilon>0$, there is a positive integer $g(\epsilon)$ such that every string graph on $n$ vertices with girth at least $g(\epsilon)$ has at most $(1+\epsilon) n$ edges.

In particular, this theorem implies that there exists a positive integer $g_{0}$ such that every string graph with girth at least $g_{0}$ has chromatic number at most 3 . It would be interesting to determine the smallest such integer $g_{0}$.

We mention another application of Theorem 1.1. The bandwidth of a graph $G$ with $n$ vertices is the minimum $b$ such that there is a labeling of the vertices of $G$ by $1, \ldots, n$ so that the labels of adjacent vertices differ by at most $b$. Chung [6] showed that every tree with $n$ vertices and maximum degree $\Delta$ has bandwidth at most $O\left(n / \log _{\Delta} n\right)$. Böttcher, Pruessmann, Taraz, and Würfl [4] used the separator theorem for planar graphs to extend this result to show that every planar graph with $n$ vertices and maximum degree $\Delta$ has bandwidth at most $O\left(n / \log _{\Delta} n\right)$. Replacing the separator theorem for planar graphs by Theorem 1.1 in the proof of this result, we obtain the following extension to all string graphs with a forbidden bipartite subgraph.

Corollary 1.7 Every $K_{t, t}$-free string graphs with $n$ vertices and maximum degree $\Delta$ has bandwidth at most $c_{t} n / \log _{\Delta} n$, where $c_{t}$ only depends on $t$.

The proof of our separator theorem, Theorem 1.1, is given in Section 2. In Section 3, we quickly deduce a qualitative version of Theorem 1.3, which states that $K_{t, t}$-free string graphs with $n$ vertices have at most $c_{t} n$ edges. The proof of Theorem 1.3 is given in Section 4. In Section 5, we prove Theorem 1.5 and a similar result for intersection graphs of convex sets in the plane. In Section 6, we deduce Theorem 1.6 and two other results that can be obtained similarly. Throughout the paper, we systematically omit floor and ceiling signs, whenever they are not crucial for the sake of clarity of the presentation. 


\section{Proof of Theorem 1.1}

The bisection width $b_{w}(G)$ of a graph $G=(V, E)$ with respect to a weight function $w$ is the least integer for which there is a partition $V=V_{1} \cup V_{2}$ such that $w\left(V_{1}\right), w\left(V_{2}\right) \leq 2 / 3$ and the number of edges between $V_{1}$ and $V_{2}$ is $b_{w}(G)$. If $w$ is the "homogeneous" weight function $w(v)=\frac{1}{|V|}$ for all $v \in V$, for simplicity we write $b(G)$ for $b_{w}(G)$.

A topological graph is a graph drawn in the plane with vertices as points and edges as curves connecting its vertices. These curves are disjoint from the vertices except for their endpoints. The pair-crossing number $\operatorname{pcr}(G)$ of a graph $G$ is the smallest number of pairs of edges that intersect in a drawing of $G$ in the plane. For any graph $G$, let $\operatorname{ssqd}(G)=\sum_{v \in V(G)}(\operatorname{deg}(v))^{2}$. We will use the following result of Kolman and Matoušek [17].

Theorem 2.1 (Kolman and Matoušek [17]) Every graph $G$ on $n$ vertices satisfies

$$
b(G) \leq c \log n(\sqrt{\operatorname{pcr}(G)}+\sqrt{\operatorname{ssqd}(G)}),
$$

where $c$ is an absolute constant.

By iterating the previous theorem, we obtain the following result.

Theorem 2.2 Let $G$ be a topological graph with $n$ vertices and maximum degree $d$, and assume that every edge of $G$ intersects at most $D$ other edges. For any weight function w, we have

$$
b_{w}(G)=O((\sqrt{d D}+d) \sqrt{n} \log n) .
$$

Proof. The maximum degree is $d$, so that the number of edges of $G$ is at most $d n / 2$. Since each edge of $G$ intersects at most $D$ other edges, the pair-crossing number of $G$ is at most $\frac{d n}{2} \frac{D}{2}=d D n / 4$.

Let $A_{0}$ denote the vertex set of $G$. By Theorem 2.1, there is a partition $A_{0}=A_{1} \cup B_{1}$ such that $\left|A_{1}\right|,\left|B_{1}\right| \leq \frac{2}{3} n$, and the number of edges with one vertex in $A_{1}$ and the other in $B_{1}$ is at most

$$
c \log n(\sqrt{\operatorname{pcr}(G)}+\sqrt{\operatorname{ssqd}(G)}) \leq c \log n\left(\sqrt{d D n / 4}+\sqrt{d^{2} n}\right) .
$$

Without loss of generality, we may assume that $w\left(A_{1}\right) \geq w\left(B_{1}\right)$.

After $i$ iterations, we have a vertex subset $A_{i}$ with at most $\left(\frac{2}{3}\right)^{i} n$ vertices. By Theorem 2.1 applied to the subgraph $G\left[A_{i}\right]$ of $G$ induced by $A_{i}$, there is a partition $A_{i}=A_{i+1} \cup B_{i+1}$ such that $\left|A_{i+1}\right|,\left|B_{i+1}\right| \leq \frac{2}{3}\left|A_{i}\right| \leq\left(\frac{2}{3}\right)^{i+1} n$, and the number of edges with one vertex in $A_{i+1}$ and the other in $B_{i+1}$ is at most

$$
\begin{aligned}
c \log n\left(\sqrt{\operatorname{pcr}\left(G\left[A_{i}\right]\right)}+\sqrt{\operatorname{ssqd}\left(G\left[A_{i}\right]\right)}\right) & \leq c \log \left(\left(\frac{2}{3}\right)^{i} n\right)\left(\sqrt{d D\left(\frac{2}{3}\right)^{i} n / 4}+\sqrt{d^{2}\left(\frac{2}{3}\right)^{i} n}\right) \\
& \leq\left(\frac{2}{3}\right)^{i / 2} c(\sqrt{d D}+d) \sqrt{n} \log n .
\end{aligned}
$$

Without loss of generality, we may assume that $w\left(A_{i+1}\right) \geq w\left(B_{i+1}\right)$. 
We stop the iterative process with $i_{0}$ if $w\left(A_{i_{0}}\right) \leq \frac{2}{3}$. Since $w\left(A_{i_{0}}\right)+w\left(B_{i_{0}}\right)=w\left(A_{i_{0}-1}\right)>2 / 3$, we have $1 / 3<w\left(A_{i_{0}}\right) \leq 2 / 3$. Let $X=A_{i_{0}}$ and $Y=A_{0} \backslash A_{i_{0}}=B_{1} \cup \ldots \cup B_{i_{0}}$. By construction, the number of edges of $G$ with one vertex in $X$ and the other vertex in $Y$ is less than

$$
\sum_{i=0}^{\infty}\left(\frac{2}{3}\right)^{i / 2} c(\sqrt{d D}+d) \sqrt{n} \log n \leq 6 c(\sqrt{d D}+d) \sqrt{n} \log n .
$$

Thus, $A_{0}=X \cup Y$ is a partition of the vertex set demonstrating that the bisection width of $G$ with respect to $w$ is $O((\sqrt{d D}+d) \sqrt{n} \log n)$.

We next prove a separator theorem for string graphs of maximum degree $\Delta$.

Theorem 2.3 Let $C$ be a collection of curves in the plane whose intersection graph $G$ has $m$ edges and maximum degree $\Delta$, and let $w$ be a weight function on $G$. Then $G$ has a separator of size $O(\Delta \sqrt{m} \log m)$ with respect to $w$.

Proof. By slightly perturbing the curves in $C$, if necessary, we can assume that no three curves in $C$ share a point in common. We may also assume without loss of generality that every element of $C$ intersects at least one other element.

For each pair of intersecting curves, pick a point of intersection, and let $P$ be the set of these $m$ points. Define the topological graph $T$ on the vertex set $P$ by connecting a pair of points of $P$ with an edge if and only if they are consecutive points of $P$ along a curve in $C$. The number of vertices of $T$ is $m$. Since no three curves in $C$ have a point in common, the maximum degree of the vertices of $T$ is at most four. Each curve in $C$ gives rise to a path in the topological graph $T$ with at most $\Delta$ vertices and at most $\Delta-1$ edges. Since each curve in $C$ intersects at most $\Delta$ other curves, each edge of $T$ crosses at most $\Delta$ curves, besides the one it is contained in. Each of these at most $\Delta$ curves contains at most $\Delta-1$ edges of $T$, therefore, each edge of $T$ intersects altogether at most $\Delta(\Delta-1)<\Delta^{2}$ other edges.

For any $\gamma \in C$, let $d(\gamma)$ denote the number of points of $P$ on $\gamma$, i.e., the number of curves in $C$ that intersect $\gamma$. To each vertex $v$ of $T$ that is the intersection of two elements $\gamma_{1}, \gamma_{2} \in C$, assign the weight

$$
w^{\prime}(v)=\frac{w\left(\gamma_{1}\right)}{d\left(\gamma_{1}\right)}+\frac{w\left(\gamma_{2}\right)}{d\left(\gamma_{2}\right)}
$$

Notice that $w^{\prime}(P)=w(C)=1$.

We now apply Theorem 2.2 to the topological graph $T$ and to the weight function $w^{\prime}$. Recall that $T$ has $m$ vertices, maximum degree at most four, and every edge intersects at most $\Delta^{2}$ other edges. So there is a partition $P=P_{1} \cup P_{2}$ with $w^{\prime}\left(P_{1}\right), w^{\prime}\left(P_{2}\right) \leq 2 / 3$ and the number of edges with one vertex in $P_{1}$, and the other in $P_{2}$ is

$$
O\left(\left(\Delta^{2} m\right)^{1 / 2} \log m\right)=O\left(\Delta m^{1 / 2} \log m\right) .
$$

Let $C_{0}$ consist of those curves in $C$ that contain an edge of the topological graph $T$ with one vertex in $P_{1}$ and the other in $P_{2}$. There are $O\left(\Delta m^{1 / 2} \log m\right)$ such edges, therefore we have $\left|C_{0}\right|=O\left(\Delta m^{1 / 2} \log m\right)$. 
For $i \in\{1,2\}$, let $C_{i}$ consist of those curves of $C$ all of whose intersection points in $P$ belong to $P_{i}$. Note that, by construction, $w\left(C_{i}\right) \leq w^{\prime}\left(P_{i}\right) \leq 2 / 3$. We claim that $C=C_{0} \cup C_{1} \cup C_{2}$ is a partition of $C$, and hence $C_{0}$ is a separator for $G$ with respect to $w$.

Each curve in $C_{0}$ contains an edge with one endpoint in $P_{1}$ and the other in $P_{2}$. Thus, $C_{0}$ is disjoint from $C_{1}$ and from $C_{2}$. To show that $C_{1} \cap C_{2}=\emptyset$, it is enough to notice that any curve $\gamma$ of $C$ which contains a point in $P_{1}$ and one in $P_{2}$ must belong to $C_{0}$, because it gives rise to a path in $T$, therefore it must contain an edge from $P_{1}$ to $P_{2}$. Thus, $C=C_{0} \cup C_{1} \cup C_{2}$ is a partition of $C$, and $C_{0}$ is a separator with respect to $w$, of the desired size.

We are now ready to prove Theorem 1.1, which states that for every string graph $G$ with $m$ edges and for every weight function $w$ for $G$, there is a separator of size $O\left(m^{3 / 4} \sqrt{\log m}\right)$ with respect to $w$. Proof of Theorem 1.1. Let $\Delta=m^{1 / 4} / \sqrt{\log m}$. Let $C$ be a collection of curves in the plane whose intersection graph is the string graph $G$. Let $C^{\prime}$ denote the set of all curves in $C$, the degree of which in $G$ is at least $\Delta$. We have $\left|C^{\prime}\right| \leq 2 m / \Delta=2 m^{3 / 4} \sqrt{\log m}$. In the subgraph $G^{\prime}$ of $G$ induced by the remaining vertices, the maximum degree is at most $\Delta$. Applying Theorem 2.3 to this graph and to the weight function $w$ restricted to $C \backslash C^{\prime}$, we conclude that there is a separator $C^{\prime \prime}$ for $G^{\prime}$ of size $O(\Delta \sqrt{m} \log m)$. Hence, $C^{\prime} \cup C^{\prime \prime}$ is a separator for $G$ of size $O\left(m^{3 / 4} \sqrt{\log m}\right)$, completing the proof.

\section{$3 \quad H$-free string graphs have linearly many edges}

In this section, we show how Theorem 1.3 can be deduced in a few lines from our separator theorem, Theorem 1.1, if we pay no attention to the dependence of the constant coefficient of $n$ on $t$.

A weaker version of Theorem 1.3, established in [29], states that every $K_{t, t}$-free string graph on $n$ vertices has at most $n \log ^{c t} n$ edges. Combining this theorem with Theorem 1.1, we obtain the following corollary.

Corollary 3.1 For every $K_{t, t}$-free string graph $G$ on $n$ vertices and for every weight function $w$ for $G$, there is a separator of size $n^{3 / 4} \log ^{c t} n$ with respect to $w$, where $c_{t}$ is a constant depending only on $t$.

A family of graphs is hereditary if it is closed under taking induced subgraphs. The following lemma of Lipton, Rose, and Tarjan [23] shows that if all members of a hereditary family of graphs have small separators, then the number of edges of these graphs is at most linear in the number of vertices. Another proof with a slightly better bound can be found in [10].

Lemma 3.2 (Lipton, Rose, Tarjan [23]) Let $\epsilon>0$, and let $F$ be a hereditary family of graphs such that every member of $F$ with $n$ vertices has a separator of size $O\left(n /(\log n)^{1+\epsilon}\right)$. Then every graph in $F$ on $n$ vertices has at most $c_{F} n$ edges, where $c_{F}$ is a suitable constant.

Clearly, the family of $K_{t, t}$-free string graphs is hereditary. Therefore, Corollary 3.1 combined with Lemma 3.2 immediately implies that every $K_{t, t}$-free string graph on $n$ vertices have at most $c_{t} n$ edges, where $c_{t}$ only depends on $t$. 


\section{Proof of Theorem 1.3}

The aim of this section is to prove Theorem 1.3. (A proof disregarding the dependence of the constant on $t$ was given in Section 3.)

The first ingredient of the proof of Theorem 1.3 is a weaker upper bound on the number of edges of a $K_{t, t}$-free string graph on $n$ vertices. Pach and Sharir proved that every $K_{t, t}$-free string graph on $n$ vertices has at most $n \log ^{c_{t}} n$ edges. Their proof shows that we may take $c_{t}=2^{c t}$ for some absolute constant $c$. We first show how to modify their proof technique, in combination with other extremal results on string graphs, to show that the result also holds with $c_{t}=c \log t$.

Lemma 4.1 Every string graph $G$ with $n$ vertices and at least $n \log ^{c_{1} \log t} n$ edges has $K_{t, t}$ as a subgraph, where $c_{1}$ is an absolute constant.

To prove this lemma, we need the following two auxiliary results. The first of these results, from [12], shows that every $n$-vertex string graph with positive constant edge density contains a balanced complete bipartite graph with $\Omega(n / \log n)$ vertices.

Lemma 4.2 ([12]) Every string graph with $n$ vertices and $\epsilon n^{2}$ edges has $K_{t, t}$ as a subgraph with $t=\epsilon^{c_{3}} n / \log n$ for some absolute constant $c_{3}$.

The following lemma guarantees that topological graphs on $n$ vertices with sufficiently many edges contain $s$ pairwise crossing edges with distinct vertices. The same result was proved in [11], except that the $s$ pairwise crossing edges were allowed to share endpoints. As we will need the slightly stronger version for the proof of Theorem 1.3, we include its proof here.

Lemma 4.3 There is an absolute constant $c_{2}$ such that every topological graph with $n$ vertices and at least $n(\log n)^{c_{2} \log s}$ edges has s pairwise crossing edges with distinct vertices.

We will use the following lemma, which shows that for every graph $G$ with $n$ vertices and $m \gg n$ edges, almost all induced subgraphs of $G$ have roughly $m / 4$ edges.

Lemma 4.4 Let $G$ be a graph with $n$ vertices and $m \geq n$ edges. Let $H$ be an induced subgraph of $G$ taken uniformly at random and $X$ be the random variable denoting number of edges of $H$. For every $\lambda>0$,

$$
\mathbb{P}[|X-m / 4| \geq \lambda \sqrt{m n} / 2] \leq 1 / \lambda^{2}
$$

Proof. Let $d_{1}, \ldots, d_{n}$ be the degree sequence of $G$, so $m=\frac{1}{2} \sum_{i=1}^{n} d_{i}$. Let $\sigma=\frac{1}{4}\left(3 m+2 \sum_{i=1}^{n}\left(\begin{array}{c}d_{i} \\ 2\end{array}\right)\right)^{1 / 2}$. We have

$$
\sigma^{2}=\frac{1}{16}\left(3 m+2 \sum_{i=1}^{n}\left(\begin{array}{c}
d_{i} \\
2
\end{array}\right)\right)=\frac{1}{16}\left(m+\sum_{i=1}^{n} d_{i}^{2}\right) \leq \frac{1}{16}\left(m+\frac{2 m}{n} n^{2}\right)=\frac{1}{16}(m+2 m n) \leq m n / 4,
$$

where the first inequality uses the convexity of the function $f(y)=y^{2}$ together with the inequalities $0 \leq d_{i} \leq n$ and the equation $\sum_{i=1}^{n} d_{i}=2 m$. Taking square roots, we obtain $\sigma \leq \sqrt{m n} / 2$. 
The proof uses the Second Moment Method (see, e.g., Section 4 of [2]). Namely, we show that the expected value and standard deviation of the random variable $X$ are $m / 4$ and $\sigma$, respectively. The desired inequality is just Chebyschev's inequality using the above upper bound on $\sigma$.

We pick a vertex to be in $H$ with probability $1 / 2$ independently of the other vertices. For each edge $e$ of $G$, let $X_{e}$ be the indicator random variable of the event that $e$ is an edge of $H$. That is, $X_{e}=1$ if $e$ is an edge of $H$ and $X_{e}=0$ otherwise. We have $\mathbb{E}\left[X_{e}\right]=1 / 4$, and by linearity of expectation, $\mathbb{E}[X]=\sum_{e \in E(G)} \mathbb{E}\left[X_{e}\right]=m / 4$.

Since $X=\sum_{e \in E(G)} X_{e}$, we have

$$
\operatorname{Var}[X]=\sum_{e \in E(G)} \operatorname{Var}\left[X_{e}\right]+\sum_{e \neq e^{\prime}} \operatorname{Cov}\left[X_{e}, X_{e^{\prime}}\right]
$$

where the variance is defined by $\operatorname{Var}[X]=\mathbb{E}\left[X^{2}\right]-\mathbb{E}[X]^{2}$ and the covariance is defined by $\operatorname{Cov}\left[X_{e}, X_{e^{\prime}}\right]=$ $\mathbb{E}\left[X_{e} X_{e^{\prime}}\right]-\mathbb{E}\left[X_{e}\right] \mathbb{E}\left[X_{e^{\prime}}\right]$. Since $X_{e}$ is an indicator random variable, we have $\operatorname{Var}\left[X_{e}\right]=\mathbb{E}\left[X_{e}^{2}\right]-\mathbb{E}\left[X_{e}\right]^{2}=$ $\mathbb{E}\left[X_{e}\right]-\mathbb{E}\left[X_{e}\right]^{2}=3 / 16$. The covariance of independent variables is 0 . In particular, if $e$ and $e^{\prime}$ do not share a vertex, then $\operatorname{Cov}\left[X_{e}, X_{e^{\prime}}\right]=0$. Note that if $e$ and $e^{\prime}$ share an edge, then $X_{e} X_{e^{\prime}}=1$ if and only if the three vertices of $e$ or $e^{\prime}$ are all vertices of $H$. Hence, in this case, $\mathbb{E}\left[X_{e} X_{e^{\prime}}\right]=1 / 8$ and $\operatorname{Cov}\left[X_{e}, X_{e^{\prime}}\right]=\mathbb{E}\left[X_{e} X_{e^{\prime}}\right]-\mathbb{E}\left[X_{e}\right] \mathbb{E}\left[X_{e^{\prime}}\right]=1 / 8-1 / 16=1 / 16$. The number of pairs of distinct edges that share an edge, by counting over the vertex in common of the two edges, is precisely $\sum_{i=1}^{n}\left(\begin{array}{c}d_{i} \\ 2\end{array}\right)$. Putting this altogether and using linearity of expectation,

$$
\operatorname{Var}[X]=\sum_{e \in E(G)} \operatorname{Var}\left[X_{e}\right]+\sum_{e \neq e^{\prime}} \operatorname{Cov}\left[X_{e}, X_{e^{\prime}}\right]=\frac{3}{16} m+\frac{2}{16} \sum_{i=1}^{n}\left(\begin{array}{c}
d_{i} \\
2
\end{array}\right) .
$$

The standard deviation of $X$ is therefore $\sqrt{\operatorname{Var}[X]}=\sigma$, which completes the proof.

The next statement is an easy consequence of Lemma 4.4.

Lemma 4.5 Let $G_{1}$ and $G_{2}$ be graphs on the same vertex set $V$ of cardinality $n$ and denote the number of edges of $G_{i}$ by $m_{i}$. If $m_{i} \geq 64 n$ for $i=1,2$, then there is a partition $V=V_{1} \cup V_{2}$ such that the induced subgraph of $G_{i}$ by $V_{i}$ has at least $m_{i} / 8$ edges, for $i=1,2$.

Proof. Let $\lambda=2$. Pick $V_{1} \subset V$ uniformly at random, and let $V_{2}=V \backslash V_{1}$. For each $i=1,2$, since $\lambda \sqrt{m_{i} n} / 2 \leq m_{i} / 8$, Lemma 4.4 implies that the probability that the number of edges of the induced subgraph of $G_{i}$ by $V_{i}$ is less than $m_{i} / 8$ is at most $1 / 4$. Hence, with probability at least $1 / 2$, for each $i=1,2$, the number of edges of the induced subgraph of $G_{i}$ by $V_{i}$ is at least $m_{i} / 8$. Since this event occurs with positive probability, there is a partition $V=V_{1} \cup V_{2}$ such that the number of edges of the induced subgraph of $G_{i}$ by $V_{i}$ is at least $m_{i} / 8$, for $i=1,2$.

We have now established the necessary lemmas to present the proof of Lemma 4.3. Our proof is similar to the proof of Theorem 11 from [11], but it also guarantees that the $s$ pairwise crossing edges have distinct vertices. 
Proof of Lemma 4.3: Let $P(n, s)$ denote the maximum number of edges of a topological graph on $n$ vertices with no $s$ pairwise crossing edges with distinct vertices. We will prove by induction on $n$ and $s$ the upper bound

$$
P(n, s)<n(\log n)^{c_{2} \log s},
$$

which implies Lemma 4.3. Note the simple bounds $P(n, s) \leq\left(\begin{array}{l}n \\ 2\end{array}\right)$ and $P(n, 1)=0$, which are our base cases. The induction hypothesis is that if $s^{\prime} \leq s$ and $n^{\prime} \leq n$ and $\left(n^{\prime}, s^{\prime}\right) \neq(n, s)$, then $P\left(n^{\prime}, s^{\prime}\right)<$ $n^{\prime}\left(\log n^{\prime}\right)^{c_{2} \log s^{\prime}}$. Let $G=(V, E)$ be a topological graph with $n$ vertices, $m=P(n, s)$ edges, and no $s$ pairwise crossing edges with distinct vertices. Let $F$ be the intersection graph of the edges of $G$, and $x$ denote the number of edges of $F$, i.e., $G$ has $x$ pairs of crossing edges. Let $y=100 c^{2} \log ^{4} n$, where $c$ is the absolute constant from Lemma 2.1.

Case 1: $x<m^{2} / y$. Note that $x$ is an upper bound on the pair-crossing number of $G$. By Theorem 2.1, there is a partition $V=V_{1} \cup V_{2}$ such that $\left|V_{1}\right|,\left|V_{2}\right| \leq \frac{2}{3}|V|$ and the number of edges with one vertex in $V_{1}$ and the other in $V_{2}$ satisfies

$$
e\left(V_{1}, V_{2}\right)=b(G) \leq c \log n(\sqrt{\operatorname{pcr}(G)}+\sqrt{\operatorname{ssqd}(G)}) .
$$

Note that by the convexity of the function $f(z)=z^{2}$, the degrees of the vertices in $G$ lie between 0 and $n$, and the sum of the degrees of the vertices in $G$ is $2 m$, so that $\operatorname{ssqd}(G) \leq \frac{2 m}{n} n^{2}=2 m n$. If $m<2 n y$, then we are done. Thus, we may assume that $m \geq 2 n y$ and it follows that $\sqrt{x}+\sqrt{\operatorname{ssqd}(G)} \leq 2 m y^{-1 / 2}$. Hence, $e\left(V_{1}, V_{2}\right) \leq c \log n \cdot 2 m y^{-1 / 2} \leq \frac{m}{5 \log n}$. For $i=1,2$, the subgraph of $G$ induced by $V_{i}$ also has no $s$ pairwise crossing edges with distinct vertices. Hence,

$$
m \leq P\left(\left|V_{1}\right|, s\right)+P\left(\left|V_{2}\right|, s\right)+\frac{m}{5 \log n} .
$$

Using the induction hypothesis, the inequality $\left|V_{1}\right|,\left|V_{2}\right| \leq 2 n / 3$, and that $c_{2}$ is a sufficiently large constant, we have

$$
\begin{aligned}
P(n, s) & =m \leq\left(1-\frac{1}{5 \log n}\right)^{-1}\left(P\left(\left|V_{1}\right|, s\right)+P\left(\left|V_{2}\right|, s\right)\right) \\
& \leq\left(1-\frac{1}{5 \log n}\right)^{-1}\left(\left|V_{1}\right|\left(\log \left|V_{1}\right|\right)^{c_{2} \log s}+\left|V_{2}\right|\left(\log \left|V_{2}\right|\right)^{c_{2} \log s}\right) \\
& \leq\left(1-\frac{1}{5 \log n}\right)^{-1} n(\log (2 n / 3))^{c_{2} \log s}<n(\log n)^{c_{2} \log s},
\end{aligned}
$$

which completes this case.

Case 2: $x \geq m^{2} / y$. So $F$, the intersection graph of the edges of $G$, has at least $m^{2} / y$ edges. Since $F$ is a string graph, Lemma 4.2 implies that there is an absolute constant $c_{3}$ such that $F$ contains $K_{t, t}$ as a subgraph with

$$
t=y^{-c_{3}} m / \log m \geq 100^{-c_{3}} c^{-2 c_{3}}(\log n)^{-4 c_{3}} m / \log m \geq m(\log n)^{-c^{\prime}},
$$

for some absolute constants $c^{\prime}$. Hence, there are two edge subsets $E_{1}, E_{2}$ of $G$, each of size at least $t$, such that every edge in $E_{1}$ crosses every edge in $E_{2}$. Applying Lemma 4.5, there are edge subsets $E_{1}^{\prime} \subset E_{1}$ and $E_{2}^{\prime} \subset E_{2}$, each of cardinality at least $t / 8$, such that the vertices of the edges in $E_{1}^{\prime}$ are 
distinct from the vertices in $E_{2}^{\prime}$. Since $G$ has no $s$ pairwise crossing edges with distinct vertices, there is $i \in\{1,2\}$ such that $E_{i}^{\prime}$ does not contain $s / 2$ pairwise crossing edges. Hence,

$$
m(\log n)^{-c^{\prime}} / 8 \leq t / 8 \leq\left|E_{i}^{\prime}\right| \leq P(n,\lceil s / 2\rceil) \leq n(\log n)^{c_{2} \log \lceil s / 2\rceil},
$$

which implies $m \leq n(\log n)^{c_{2} \log s}$ since $c_{2}$ was chosen to be a sufficiently large absolute constant. This completes the proof.

Having gathered the required lemmas, we now prove Lemma 4.1. A Jordan region is a closed region of the plane, bounded by a simple closed Jordan curve. In other words, a Jordan region is homeomorphic to the closed unit disk.

Proof of Lemma 4.1. Let $s$ be the smallest positive integer such that $(1 / 16)^{c_{3}}(2 s) / \log 2 s \geq t$, where $c_{3}$ is the absolute constant from Lemma 4.2. Let $c_{t}=c_{2} \log s=O(\log t)$, where $c_{2}$ is the absolute constant from Lemma 4.3.

Suppose $G$ is a string graph with $n$ vertices and at least $n(\log n)^{c_{t}}$ edges. Let $\mathcal{C}=\left\{C_{1}, \ldots, C_{n}\right\}$ be a collection of $n$ Jordan regions whose intersection graph is the string graph $G$ and which have the property that any two intersecting Jordan regions in $\mathcal{C}$ intersect in their interiors (it is easy to see that, by slightly fattening compact connected sets, every string graph is the intersection graph of such a collection of Jordan regions). Fix distinct points $p_{i}$ in the interior of $C_{i}$ for $i=1, \ldots, n$. For each intersecting pair $C_{i}, C_{j} \in \mathcal{C}$ with $i<j$, let $p_{i j}$ be a point in $C_{i} \cup C_{j}$ such that all the points in $\left\{p_{1}, \ldots, p_{n}\right\} \cup\left\{p_{i j}: C_{i} \cup C_{j} \neq \emptyset\right\}$ are distinct, and let $\gamma_{i j}$ be a simple (nonintersecting) curve contained in $C_{i} \cup C_{j}$ such that

1. $\gamma_{i j}$ has endpoints $p_{i}$ and $p_{j}$;

2. $\gamma_{i j}$ is disjoint from all other $p_{\ell}$;

3. $\gamma_{i j}$ can be split into two subcurves $\gamma_{i j}^{0}$ and $\gamma_{i j}^{1}$ such that $\gamma_{i j}^{0}$ is contained in $C_{i}$ and has endpoints $p_{i}$ and $p_{i j}$ and $\gamma_{i j}^{1}$ is contained in $C_{j}$ and has endpoints $p_{i j}$ and $p_{j}$.

The points $\left\{p_{1}, \ldots, p_{n}\right\}$ are the vertex set and curves $\left\{\gamma_{i j}: C_{i} \cap C_{j} \neq \emptyset\right\}$ are the edge set of a topological graph $T$ with $n$ vertices and at least $n(\log n)^{c_{t}}$ edges. Since $c_{t}=c_{2} \log s$, by Lemma 4.3, there are at least $s$ pairwise intersecting edges in $T$ with distinct vertices. Each edge consists of two subcurves and these $2 s$ subcurves have at least $\left(\begin{array}{l}s \\ 2\end{array}\right) \geq \frac{1}{16}(2 s)^{2}$ intersecting pairs. By Lemma 4.2 , the intersection graph of these $2 s$ subcurves contains $K_{h, h}$ with $h=(1 / 16)^{c_{3}}(2 s) / \log 2 s \geq t$. It follows from the construction that $G$ contains $K_{t, t}$.

The second ingredient of the proof of Theorem 1.3 is our separator theorem, Theorem 1.1, which, together with Lemma 4.1, implies that every $K_{t, t}$-free string graph with $m<n(\log n)^{c_{t}}$ edges has a separator of size $O\left(m^{3 / 4} \sqrt{\log m}\right)<O\left(n^{3 / 4} \log ^{3 c_{t} / 4+1 / 2} n\right)$. Thus, for $n_{0}=2^{O\left(c_{t} \log c_{t}\right)} \leq t^{c^{\prime} \log \log t}$ (where $c^{\prime}$ is an absolute constant), every $K_{t, t}$-free string graph with $n \geq n_{0}$ vertices has a separator of size $n^{7 / 8}$. This fact, together with the following lemma from [10] (which is a more precise version of Lemma 3.2), immediately implies Theorem 1.3. 
Lemma 4.6 ([10]) Let $\phi(n)$ be a monotone decreasing nonnegative function defined on the set of positive integers, and let $n_{0}$ and $C$ be positive integers such that

$$
\phi\left(n_{0}\right) \leq \frac{1}{12} \text { and } \prod_{i=0}^{\infty}\left(1+\phi\left(\left\lceil(4 / 3)^{i} n_{0}\right\rceil\right)\right) \leq C .
$$

If $F$ is an $n \phi(n)$-separable hereditary family of graphs, then every graph in $F$ on $n \geq n_{0}$ vertices has fewer than $\frac{C n_{0}}{2} n$ edges.

\section{$5 \quad$ Stronger versions of Theorem 1.3}

First we establish Theorem 1.5, that is, we show how to improve considerably Theorem 1.3 for intersection graphs of collections of curves, in which every pair of curves intersect in at most a fixed constant number $k$ of points.

In [10], we proved that the intersection graph of a collection of curves with $x$ crossings has a separator of size $O(\sqrt{x})$. If each pair in a collection of curves intersect in at most $k$ points, then the number $m$ of edges of the intersection graph is at least $x / k$, and we obtain a separator of size $O(\sqrt{\mathrm{km}})$. In [13], the following result was established, which is an analogue of Lemma 4.2 for families of curves in which each pair intersects in at most a constant $k$ number of points.

Lemma 5.1 Let $G$ be the intersection graph of a collection of $n$ curves in the plane, any pair of which intersect in at most $k$ points. If $G$ has at least $\epsilon n^{2}$ edges, then it contains a complete bipartite subgraph $K_{t, t}$ with $t \geq c_{k} \epsilon^{c} n$, where $c$ is an absolute constant and $c_{k}$ is a constant that only depends on $k$.

We now have the necessary tools to prove Theorem 1.5. This theorem states that for positive integers $k$ and $t$, there exists a constant $C_{k}$ depending only on $k$, such that any $K_{t, t}$-free intersection graph $G$ of $n$ curves in the plane, no pair of which have more than $k$ points in common, has at most $C_{k}$ tn edges.

Proof of Theorem 1.5. Suppose that $G$ has $\epsilon n^{2}$ edges. By Lemma 5.1, we have $t \geq c_{k} \epsilon^{c} n$, that is, $\epsilon \leq\left(c_{k}^{-1} \frac{t}{n}\right)^{1 / c}$. Thus, according to the separator lemma mentioned above, $G$ has a separator of size $O(\sqrt{k m})<O\left(\sqrt{k\left(c_{k}^{-1} \frac{t}{n}\right)^{1 / c} \cdot n^{2}}\right)<c_{k}^{\prime}(t / n)^{c_{1}} n$, where $c_{1}=1 /(2 c)>0$ and $c_{k}^{\prime}$ only depends on $k$.

Letting $\phi(n)=c_{k}^{\prime}(t / n)^{c_{1}}$ and $n_{0}=\left(12 c_{k}^{\prime}\right)^{-1 / c_{1}} t$, Lemma 4.6 implies that $G$ has at most $C_{k} t n$ edges for some constant $C_{k}$ only depending on $k$.

We similarly prove the following result.

Theorem 5.2 Every $K_{t, t}$-free intersection graph $G$ of $n$ convex sets in the plane has $O\left(t^{3} n\right)$ edges.

Proof. Suppose that $G$ has $\epsilon n^{2}$ edges. In [14], it was shown that every intersection graph of $n$ convex sets in the plane with $\epsilon n^{2}$ edges contains a complete bipartite subgraph $K_{t, t}$ with $t \geq c \epsilon^{2} n$ for some absolute constant $c>0$. Hence, $\epsilon \leq\left(\frac{t}{c n}\right)^{1 / 2}$. A separator lemma from [11] states that every $K_{t}$-free intersection graph of convex sets in the plane with $m$ edges has a separator of size at most $c^{\prime} \sqrt{t m}$ for 
some absolute constant $c^{\prime}$. Hence, $G$ has a separator of size $c^{\prime} \sqrt{2 t m} \leq 2 c^{\prime} \sqrt{t} \epsilon^{1 / 2} n \leq 2 c^{\prime} c^{-1 / 4} t^{3 / 4} n^{3 / 4}$. Letting $\phi(n)=2 c^{\prime} c^{-1 / 4} t^{3 / 4} n^{-1 / 4}$ and $n_{0}=24^{4} c^{\prime 4} c^{-1} t^{3}$, Lemma 4.6 implies that $G$ has $O\left(t^{3} n\right)$ edges.

\section{Proof of Theorem 1.6 and related results}

Theorem 1.6 is a direct corollary of Theorem 1.1 and the following lemma, showing that all graphs of large girth, which belong to a hereditary family of graphs with small separators, are quite sparse.

Lemma 6.1 Let $\alpha>0$, and let $F$ be a hereditary family of graphs such that every member of $F$ with $n$ vertices has a separator of size $O\left(n /(\log n)^{1+\alpha}\right)$. Then for each $\epsilon>0$ there is a positive integer $g=g_{F}(\epsilon)$ such that every graph in $F$ on $n$ vertices and girth at least $g$ has at most $(1+\epsilon) n$ edges.

The aim of this section is to prove Lemma 6.1 and to discuss some of its consequences. The similarity between Lemma 6.1 and 3.2 is no coincidence; their proofs are very similar.

Before turning to the proof, we briefly outline its main idea. Consider a hereditary family $F$ of graphs, in which every graph has a small separator. We show that every graph $G$ in $F$ with $n$ vertices has an induced subgraph with at most $\frac{3}{4} n$ vertices, whose average degree is not much smaller than the average degree of $G$. We repeatedly use this fact until we find an induced subgraph of $G$ with fewer than $g$ vertices, whose average degree is not much smaller than that of $G$. But if the girth of $G$ is at least $g$, then this induced subgraph of $G$ with fewer than $g$ vertices is a forest and so has average degree less than 2. If $g$ is chosen sufficiently large, we conclude that $G$ has average degree at most $2+2 \epsilon$ and hence at most $(1+\epsilon) n$ edges.

Now we work out the details of the proof of Lemma 6.1. Given a nonnegative function $f$ defined on the set of positive integers, we say that a family $F$ of graphs is $f$-separable, if every graph in $F$ with $n$ vertices has a separator of size at most $f(n)$.

Lemma 6.2 Let $\phi(n)$ be a monotone decreasing nonnegative function defined on the set of positive integers, and let $g$ be a positive integer and $\epsilon>0$ such that

$$
\phi(g) \leq \frac{1}{12} \text { and } \prod_{i=0}^{\infty}\left(1+\phi\left(\left\lceil(4 / 3)^{i} g\right\rceil\right)\right) \leq 1+\epsilon .
$$

If $F$ is an $n \phi(n)$-separable hereditary family of graphs, then every graph in $F$ on $n$ vertices with girth at least $g$ has fewer than $(1+\epsilon) n$ edges.

Proof. Let $G_{0}=(V, E)$ be a member of the family $F$ with $n$ vertices, girth at least $g$, and average degree $d$. If $n<g$, then $G_{0}$ is a forest and hence has at most $n-1$ edges. We may therefore assume that $n \geq g$. By definition, there is a partition $V=V_{0} \cup V_{1} \cup V_{2}$ with $\left|V_{0}\right| \leq n \phi(n),\left|V_{1}\right|,\left|V_{2}\right| \leq \frac{2}{3} n$, such that no vertex in $V_{1}$ is adjacent to any vertex in $V_{2}$. 
Let $d^{\prime}$ and $d^{\prime \prime}$ denote the average degree of the vertices in the subgraphs of $G_{0}$ induced by $V_{0} \cup V_{1}$ and $V_{0} \cup V_{2}$, respectively. Every edge of $G_{0}$ is contained in at least one of these two induced subgraphs. Hence,

$$
d^{\prime}\left(\left|V_{0}\right|+\left|V_{1}\right|\right)+d^{\prime \prime}\left(\left|V_{0}\right|+\left|V_{2}\right|\right) \geq 2|E|=d|V|,
$$

so that

$$
d^{\prime} \frac{\left|V_{0}\right|+\left|V_{1}\right|}{|V|+\left|V_{0}\right|}+d^{\prime \prime} \frac{\left|V_{0}\right|+\left|V_{2}\right|}{|V|+\left|V_{0}\right|} \geq d \frac{|V|}{|V|+\left|V_{0}\right|} .
$$

Since $|V|=\left|V_{0}\right|+\left|V_{1}\right|+\left|V_{2}\right|$, then $\frac{\left|V_{0}\right|+\left|V_{1}\right|}{|V|+\left|V_{0}\right|}+\frac{\left|V_{0}\right|+\left|V_{2}\right|}{|V|+\left|V_{0}\right|}=1$ and the left hand side of the above inequality is a weighted mean of $d^{\prime}$ and $d^{\prime \prime}$. Consequently, $d^{\prime}$ or $d^{\prime \prime}$ is at least

$$
d \frac{|V|}{|V|+\left|V_{0}\right|} \geq d \frac{1}{1+\phi(n)}
$$

Suppose without loss of generality that $d^{\prime}$ is at least as large as this number, and let $G_{1}$ denote subgraph of $G$ induced by $V_{0} \cup V_{1}$. By assumption, we have that $\phi(n) \leq \frac{1}{12}$ and $\left|V_{0}\right| \leq n \phi(n)$. Therefore, $G_{1}$ has $\left|V_{0}\right|+\left|V_{1}\right| \leq \frac{1}{12} n+\frac{2}{3} n=\frac{3}{4} n$ vertices.

Proceeding like this, we find a sequence of induced subgraphs $G_{0} \supset G_{1} \supset G_{2} \supset \ldots$ with the property that, if $G_{i}$ has $n_{i} \geq g$ vertices and average degree $d_{i}$, then $G_{i+1}$ has at most $\frac{3}{4} n_{i}$ vertices and average degree at least $\frac{1}{1+\phi\left(n_{i}\right)} d_{i}$. We stop with $G_{j}$ if the number of vertices of $G_{j}$ is less than $g$.

Since $G_{j}$ is an induced subgraph of $G$, it also has girth at least $g$. The number of vertices of $G_{j}$ is less than $g$, so $G_{j}$ must be a forest and therefore has average degree less than 2 . The above argument also shows that the average degree of $G_{j}$ is at least $\frac{1}{1+\epsilon} d$, so $d<2(1+\epsilon)$, and the number of edges of $G$ is $d n / 2<(1+\epsilon) n$, completing the proof.

Taking logarithms and approximating $\ln (1+x)$ by $x$, we obtain that $\prod_{i=0}^{\infty}\left(1+\phi\left(\left\lceil(4 / 3)^{i} g\right\rceil\right)\right) \neq \infty$ if and only if $\sum_{i=0}^{\infty} \phi\left(\left\lceil(4 / 3)^{i}\right\rceil\right) \neq \infty$ if and only if $\sum_{i=0}^{\infty} \phi\left(2^{i}\right) \neq \infty$. (For a formal proof of the elementary fact that $\prod_{i=1}^{\infty}\left(1+a_{i}\right)$ with each $a_{i}>0$ converges if and only if $\sum_{i=1}^{\infty} a_{i}$ converges, see, e.g., Theorem 3 of Section 3.7 in [19].) Therefore, Lemma 6.2 has the following corollary.

Corollary 6.3 Let $F$ be an $n \phi(n)$-separable hereditary family of graphs, where $\phi(n)$ is a monotone decreasing nonnegative function such that $\sum_{i=0}^{\infty} \phi\left(2^{i}\right) \neq \infty$. Then for each $\epsilon>0$ there is $g_{F}(\epsilon)$ such that every graph in $F$ on $n$ vertices and girth at least $g$ has at most $(1+\epsilon) n$ edges.

Since $\sum_{i=1}^{\infty} 1 / i^{1+\alpha}$ converges for all $\alpha>0$, Lemma 6.1 is an immediate consequence of Corollary 6.3.

The condition that a connected graph has large girth means that the graph is locally "tree-like." In general, this local condition does not imply that the graph also has some global tree-like properties. For instance, in 1959 Erdős [8] proved that the existence of graphs with arbitrarily large girth and chromatic number. However, according to Lemma 6.1, if every member of a hereditary family $F$ of graphs has a small separator, then the condition that a connected graph in $F$ has large girth does imply that the graph is globally tree-like. Indeed, if $\epsilon<1 / 2$, then every graph in $F$ with girth at least $g_{F}(\epsilon)$ is 2-degenerate and hence has chromatic number at most 3. Furthermore, any graph $G$ in $F$ with girth at least $\max \left(g_{F}(\epsilon / 2), 2 / \epsilon\right)$ can be turned into a forest by the removal of at most $\epsilon|G|$ edges. 
We end this section by presenting two other corollaries of Lemma 6.1. The separator theorem for graphs with an excluded minor [1], together with Lemma 6.1, imply the following.

Corollary 6.4 For any $\epsilon>0$ and positive integer $t$, there exists a positive integer $g(\epsilon, t)$ such that every $K_{t}$-minor-free graph on $n$ vertices with girth at least $g(\epsilon, t)$ has at most $(1+\epsilon) n$ edges.

A well-known result of Thomassen [33] (see also Chapter 8.2 in [7]) states that for any positive integer $t$, there exists another integer $g(t)$ such that every graph with minimum degree at least 3 and girth at least $g(t)$ contains $K_{t}$ as a minor. Obviously, Corollary 6.4 implies Thomassen's result. In fact, it can be shown by a simple argument that the two statements are equivalent. In the special case of planar graphs and, more generally, for graphs with bounded genus, the statement easily follows from Euler's polyhedral formula.

The separator theorem for intersection graphs of balls in $\mathbb{R}^{d}[26]$ together with Lemma 6.1 imply

Corollary 6.5 For any $\epsilon>0$ and positive integer $d$, there exists a positive integer $g(\epsilon, d)$ such that every intersection graph of balls in $\mathbb{R}^{d}$ with girth at least $g(\epsilon, d)$ has at most $(1+\epsilon) n$ edges.

\section{Concluding remarks}

Theorem 1.3, with a much worse dependence of the coefficient of $n$ on $t$, can also be deduced from the following result of Kuhn and Osthus [22]: For any graph $H$ and any positive integer $t$, there is a constant $c(H, t)$ such that every graph with $n$ vertices and at least $c(H, t) n$ edges, which contains no induced subdivision of $H$, contains $K_{t, t}$ as a subgraph. Let $H_{0}$ be the graph obtained from the complete graph $K_{5}$ by replacing each edge by a path of length two. Using the nonplanarity of $K_{5}$, it is easy to see that no subdivision of $H_{0}$ is a string graph. Since the family of string graphs is closed under taking induced subgraphs, no string graph contains an induced subdivision of $H_{0}$. Thus, the result of Kuhn and Osthus implies that any $K_{t, t}$-free string graph on $n$ vertices has at most $c\left(H_{0}, t\right) n$ edges. However, this proof only shows that $c\left(H_{0}, t\right)<2^{2^{2^{2^{c t l} \log t}}}$, for some absolute constant $c$.

The dependence of the coefficient of $n$ on $t$ in Theorem 1.3 could be further improved if we could prove Conjecture 1.2. Indeed, Conjecture 1.2 combined with Lemmas 4.2 and 4.6 would imply the following.

Conjecture 7.1 Every $K_{t, t}$-free string graph with $n$ vertices has $O((t \log t) n)$ edges.

Conjecture 7.1, if true, would be tight up to the constant factor. According to a construction in [9] and [30], there are string graphs with $n$ vertices and $(1-o(1)) n^{2} / 2$ edges, in which the size of the largest balanced bipartite subgraph is $O(n / \log n)$.

Another consequence of Conjecture 1.2 would be that, together with Lemma 4.2, it would imply that every $K_{t}$-free string graph with $n$ vertices has chromatic number at most $(\log n)^{c \log t}$ for some absolute constant $c$. It is not even known if every triangle-free string graph with $n$ vertices has chromatic number $n^{o(1)}$.

Acknowledgment. We would like to thank Benny Sudakov for the simple proof of Lemma 4.4. 


\section{References}

[1] N. Alon, P. Seymour, and R. Thomas, A separator theorem for nonplanar graphs. J. Amer. Math. Soc. 3 (1990), 801-808.

[2] N. Alon and J. Spencer, The Probabilistic Method, 2nd ed., Wiley, 2000.

[3] B. Bollobás, Modern Graph Theory, Springer, 1998.

[4] J. Böttcher, K. P. Pruessmann, A. Taraz, and A. Würfl, Bandwidth, treewidth, separators, expansion, and universality, in: Proc. Topological. and Geometric Graph Theory (TGGT 08), Electron. Notes. Discrete Math. 31 (2008), 91-96.

[5] V. Capoyleas and J. Pach, A Turán-type theorem on chords of a convex polygon. J. Combinatorial Theory, Ser. B 56 (1992), 9-15.

[6] F. Chung, Labelings of graphs, in: Selected Topics in Graph Theory, Academic Press, San Diego (1988), 151-168.

[7] R. Diestel, Graph Theory, 2nd ed., Springer, 2000.

[8] P. Erdős, Graph theory and probability, Canad. J. Math. 11 (1959), 34-38.

[9] J. Fox, A bipartite analogue of Dilworth's theorem, Order 23 (2006), 197-209.

[10] J. Fox and J. Pach, Separator theorems and Turán-type results for planar intersection graphs. Advances in Mathematics 219 (2008), 1070-1080.

[11] J. Fox and J. Pach, Coloring $K_{k}$-free intersection graphs of geometric objects in the plane, in: Proc. 24th ACM Sympos. on Computational Geometry, ACM Press, New York (2008), 346-354.

[12] J. Fox and J. Pach, String graphs and incomparability graphs, manuscript.

[13] J. Fox, J. Pach, and C. D. Tóth, Intersection patterns of curves, J. London Math. Soc., to appear.

[14] J. Fox, J. Pach, and C. D. Tóth, Turán-type results for partial orders and intersection graphs of convex sets, Israel J. Math, to appear.

[15] J. R. Gilbert, J. P. Hutchinson, and R. E. Tarjan, A separator theorem for graphs of bounded genus. J. Algorithms 5 (1984), 391-407.

[16] P. Koebe, Kontaktprobleme der konformen Abbildung. Berichte über die Verhandlungen der Sachsischen Akademie der Wissenschaften, Leipzig, Mathematische-Physische Klasse 88 (1936), $141-164$.

[17] P. Kolman and J. Matoušek, Crossing number, pair-crossing number, and expansion. J. Combin. Theory Ser. B 92 (2004), 99-113. 
[18] A. V. Kostochka and J. Nešetřil, Coloring relatives of intervals on the plane. I. Chromatic number versus girth. European J. Combin. 19 (1998), 103-110.

[19] K. Knopp, Infinite Sequences and Series. Dover Publications New York, 1956.

[20] J. Kratochvíl and J. Matoušek, NP-hardness results for intersection graphs. Comment. Math. Univ. Carolin. 30 (1989), 761-773.

[21] J. Kratochvíl and J. Matoušek, Intersection graphs of segments. J. Combin. Theory Ser. B 62 (1994), 289-315.

[22] D. Kühn and D. Osthus, Induced subdivisions in $K_{s, s}$ free graphs of large average degree, Combinatorica 24 (2004), 287-304.

[23] R. J. Lipton, D. J. Rose, and R. E. Tarjan, Generalized nested dissection. SIAM J. Numer. Anal. 16 (1979), 346-358.

[24] R. J. Lipton and R. E. Tarjan, A separator theorem for planar graphs. SIAM J. Appl. Math. 36 (1979), 177-189.

[25] R. J. Lipton and R. E. Tarjan, Applications of a planar separator theorem. SIAM J. Comput. 9 (1980), 615-627.

[26] G. L. Miller, S.-H. Teng, W. Thurston, and S. A. Vavasis, Separators for sphere-packings and nearest neighbor graphs. J. ACM 44 (1997), 1-29.

[27] J. Pach and P. Agarwal, Combinatorial Geometry. J. Wiley, New York, 1995.

[28] J. Pach, R. Pinchasi, M. Sharir, and G. Tóth, Topological graphs with no large grids, Graphs and Combinatorics 21 (2005), 355-364.

[29] J. Pach and M. Sharir, On planar intersection graphs with forbidden subgraphs, J. Graph Theory 59 (2009), 205-214.

[30] J. Pach, G. Tóth, Comment on Fox News, Geombinatorics 15 (2006), 150-154.

[31] F. Preparata and M. Shamos, Computational geometry. An introduction. Texts and Monographs in Computer Science. Springer-Verlag, New York, 1985.

[32] R. Radoičić and G. Tóth, The discharging method in combinatorial geometry and its application to Pach-Sharir conjecture on intersection graphs, in: Proceedings of the Joint Summer Research Conference on Discrete and Computational Geometry, (J. E. Goodman, J. Pach, J. Pollack, eds.), Contemporary Mathematics, AMS 453 (2008), 319-342.

[33] C. Thomassen, Girth in graphs. J. Combin. Theory Ser. B 35 (1983), 129-141. 